\title{
IMPROVED CHEN-SMITH IMAGE CODER *
}

\author{
Eduardo M. Rubino, Henrique S. Malvar \\ Dept. de Engenharia Elétrica, Universidade de Brasilia \\ C.P. 04591, 70919-970 Brasilia, DF, BRAZIL \\ Ricardo L. de Queiroz \\ Electrical Engineering Department, University of Texas at Arlington \\ Box 19016, Arlington, TX 76019, U.S.A.
}

\begin{abstract}
An image compression system based on transform coding and zonal sampling is presented. It is based on the wellknown Chen-Smith coder, but with an improved technique for transform coefficient quantization and the replacement of the discrete cosine transform (DCT) by the lapped orthogonal transform (LOT). The system leads to signal-to-noise ratios that are from 1.5 to $2.0 \mathrm{~dB}$ above those obtained with the standard JPEG algorithm, for a wide variety of test images and compression rates.
\end{abstract}

\section{INTRODUCTION}

Image compression has always been an important area of digital signal processing. With the rapid growth of multimedia applications, image compression is now an ordinary requirement in many systems. In most systems today, the standard JPEG algorithm [1] is used. Based on threshold coding of discrete cosine transform (DCT) coefficients, the JPEG is relatively simple to implement and leads to good results. However, threshold coding leads to some noticeable artifacts, mainly at high compression rates. Furthermore, the blocking effects typical of DCT-based coding may also be present [2].

In this paper we will present an image coder that is based on the well-known Chen-Smith (CS) algorithm [3], which can be summarized by the following steps: (1) segment the image into square blocks (usually of size $8 \times 8$ ),

"This work was supported in part by the CNPq (Brazil), under grants $300.159-90$ and $610.137-92$.

$0-7803-1254-6 / 93 \$ 03.00$ ○ 1993 IEEE apply the DCT to all of them, and extract the DC coefficients; (2) classify the blocks into four equally-populated classes, according to their AC energy; (3) compute the variances of the coefficients of the blocks within the same class; (4) run a standard bit allocation routine [4] and re-estimate the coefficient variances from the bit allocation patterns; and (5) quantize the coefficients using scalar quantizers. The information that is kept for the decoder is composed of the quantized coefficients, the bit allocation patterns for the classes, an energy normalization factor, and the DC coefficients of all blocks. The advantage of the CS algorithm over the threshold coding scheme used by JPEG is that the bit allocation routine allows the prediction of the final bit rate, which is important in applications where the encoded image size in bits is prescribed.

Our improved Chen-Smith coder (ICS) is based on two modifications to the CS coder: (1) joint quantization and entropy encoding of the transform coefficients, which allows for rate allocation instead of bit allocation; and (2) replacement of the DCT by the lapped orthogonal transform (LOT) [2]. In Section II we will describe the ICS coder, and a performance comparison with the standard JPEG coder will be presented in Section III.

\section{The ICS Adaptive Transform Coder}

The block diagram of our proposed ICS image coder is shown in Fig. 1. It is quite similar to the original CS coder [3] that was described in Section I. The main difference from the CS coder is in the quantization of the transform coefficients. In the CS coder each transform coefficient $X_{i}$ is quantized with $B_{i}$ bits, which are given 


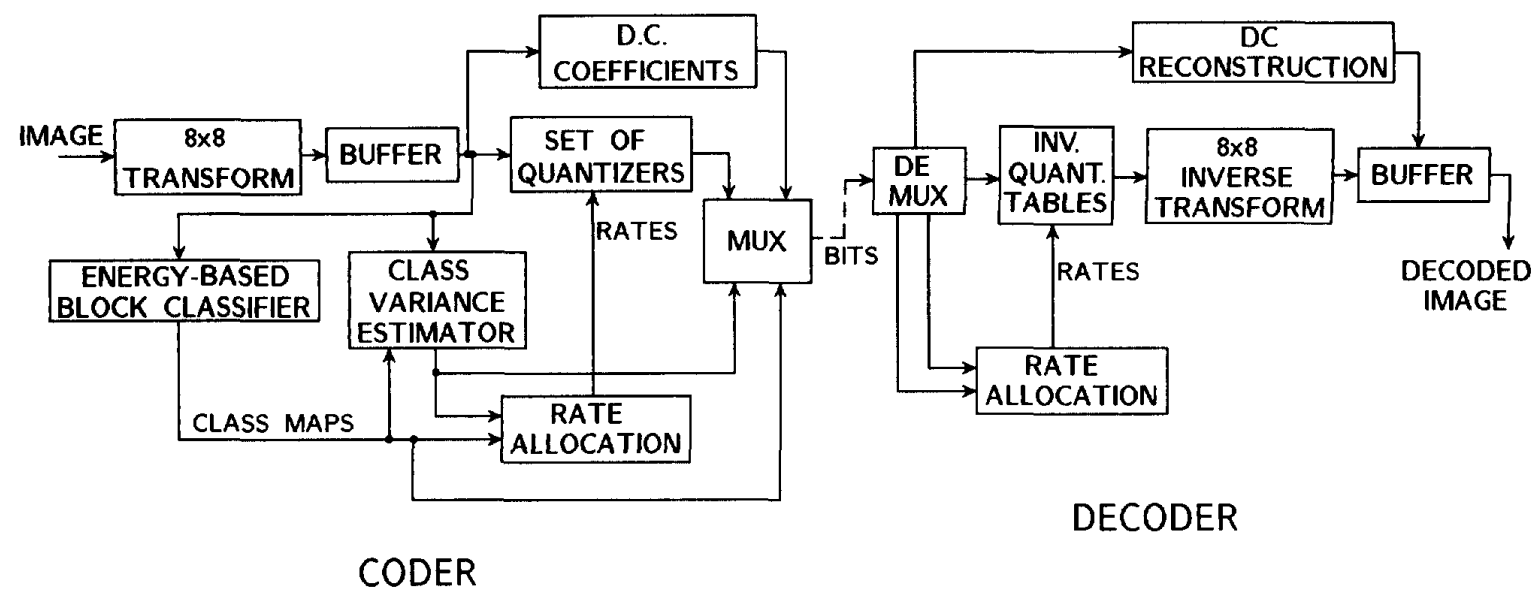

Figure 1: Block diagram of the ICS image coder/decoder.

by the optimal allocation rule

$$
B_{i}=\alpha+\frac{1}{2} \log _{2} \sigma_{i}^{2}
$$

where $\sigma_{i}^{2}$ is the variance of $X_{i}$, and $\alpha$ is a Lagrange multiplier that must be adjusted until the average of all $B_{i}$ equals the desired bit rate. If the value of $B_{i}$ predicted by (1) is negative, it is set to zero without loss of optimality [5]. Furthermore, the $B_{i}$ are generally rounded to a nearest integer, in order to simplify the implementation. This is a drawback, since quantizers with an even number of levels do not have zero as a reconstruction value, and this leads to granular noise [6].

The major feature in the ICS coder is that the $B_{i}$ in (1) are not rounded to integers, but to quarter-bits. That is, we allocate bits in integer multiples of 0.25 . In order to achieve such fractional rates, we employ a technique that we call arithmetic quantization (AQ) [7], which is based on entropy-constrained optimal quantizers with odd numbers of reconstruction levels [8]. The number of levels for quantizing $X_{i}$ must be somewhat higher than $2^{B_{i}}$. With $A Q$, the average number of bits per sample at the output is closer to the rate-distortion bound than that of the traditional Lloyd-Max quantizer.

Our rate allocation procedure is based on a slight modification of (1). Recall that, without the integer constraint and assuming ideal quantizers, the optimal bit assignment leads to [4]

$$
\gamma_{i}^{2}= \begin{cases}\sigma_{q}^{2}, & \text { if } \sigma_{i}^{2}>\sigma_{q}^{2} \\ \sigma_{i}^{2}, & \text { if } \sigma_{i}^{2} \leq \sigma_{q}^{2}\end{cases}
$$

where $\gamma_{i}^{2}$ is the error variance in the quantization of $X_{i}$, and $\sigma_{q}^{2}$ is the quantization error variance. Thus, the quantization noise is white for the coefficients that are coded, with a noise variance of $\sigma_{q}^{2}$. Using the above property, the ICS coder allocates quantization rates by

$$
B_{i}=E_{i}\left(\sigma_{q}^{2} / \sigma_{i}^{2}\right)
$$

where $E_{i}\left(\sigma^{2}\right)$ is the entropy versus error function for the optimal entropy-constrained quantizer [8] for a Gaussian probability distribution ${ }^{1}$.

The quantization variance $\sigma_{q}^{2}$ is adjusted until the average of the $B_{i}$ equals the desired rate, and each $B_{i}$ is rounded to the nearest integer multiple of 0.25 . Instead of specifying the average rate, we can also specify the average distortion $\sigma_{q}^{2}$, and (3) will lead to an average error slightly lower than $\sigma_{q}^{2}$; the total rate will be whatever is necessary to achieve the prescribed distortion. This is similar to the JPEG algorithm where a "quality factor" is specified, instead of the coded image bit rate.

We note in Fig. 1 that the rate allocation is also performed at the decoder. For that, we need to transmit

\footnotetext{
${ }^{1}$ See [9] for a justification of the use of the Gaussian p.d.f.
} 
as side information the variances of the classes. In order to keep a low overhead for this side information, we subsample by a factor of two the variance patterns, and interpolate them back before applying the rate allocation rule of (3). Besides the use of the quantization strategy described above, we employ the LOT instead of the DCT in Fig. 1. This is because the LOT is know to reduce the blocking effects typical of block transform coding $[2,5]$.

\section{Performance}

To evaluate the ICS coder, we have tested it against the JPEG coder implemented in [10], for several test images and bit rates. The ICS coder led to a slightly better picture quality than the JPEG coder in virtually all cases, and the signal-to-noise ratio (SNR) of the ICS coder was between 1.5 and $2.1 \mathrm{~dB}$ higher than that of the JPEG coder. We have considered the SNR defined as

$$
\mathrm{SNR}=10 \log _{10}\left(\frac{\sum_{i}\left(x_{i}-m\right)^{2}}{\sum_{i}\left(x_{i}-y_{i}\right)^{2}}\right)
$$

where $x_{i}$ and $y_{i}$ are the $i$ th original and reconstructed pixels, respectively, and $m$ is the average of the pixel values, i.e., the DC level of the image.

A typical example of ICS versus JPEG performance is shown in Fig. 2. At the rate of 0.7 bits/pixel, a compression ratio of over 10:1, both coders lead to quite acceptable decoded images, but a closer look with the help of the zoomed images reveals that the ICS-processed image led to fewer artifacts than the one processed by JPEG.

Besides the SNR, another important comparison criterion is computational complexity. Using a 32-bit compiler in a 486DX-33-based personal computer, the JPEG coder and decoder run in approximately two seconds each, whereas the ICS coder and decoder take three seconds to run each. In the JPEG implementation, the DCT is fully optimized with integer arithmetic, whereas the LO'T routine in our ICS coder is a floating-point implementation. After the optimization of the LOT module, we believe that the ICS running time will be approximately the same as that of the JPEG coder.

\section{CONCLUSION}

We have presented an improved Chen-Smith (ICS) image coder, based on arithmetic quantization (arithmetic encoding of entropy-constrained optimal quantizers) of LOT-transformed image coefficients. The bit allocation procedure of the classical Chen-Smith (CS) coder was replaced by our rate allocation rule. For several test images, the ICS coder outperforms the standard JPEG coder, in terms of signal-to-noise ratio, by more than $1.5 \mathrm{~dB}$. The ICS coder can be used either in rate-constrained or distortion-constrained modes, whereas the JPEG coder is distortion-constrained only. Finally, the computational complexity of the ICS algorithm is similar to that of the JPEG system.

\section{REFERENCES}

[1] G. K. Wallace, "The JPEG still picture compression standard," Commun. ACM, vol. 34, pp. 31-44, Apr. 1991.

[2] H. S. Malvar and D. H. Staelin, "The LOT: transform coding without blocking effects," IEEE Trans. Acoust., Speech, Signal Processing, vol. 37, pp. 553559, Apr. 1989.

[3] W. H. Chen and C. H. Smith, "Adaptive coding of monochrome and color images," IEEE Trans. Commun., vol. 25, pp. 1285-1292, Nov. 1977.

[4] N. S. Jayant and P. Noll, Digital Coding of Waveforms. Englewood Cliffs, NJ: Prentice-Hall, 1984.

[5] H. S. Malvar, Signal Processing with Lapped Transforms. Boston, MA: Artech House, 1992.

[6] R. J. Clarke, Transform Coding of Images. London: Academic Press, 1985.

[7] E. M. Rubino and H. S. Malvar, "Arithmetic quantization: joint quantization and entropy coding," unpublished.

[8] N. Farvardin and J. W. Modestino, "Optimum quantizer performance for a class of non-Gaussian memoryless sources," IEEE Trans. Inform. Theory, vol. 30, pp. 485-497, May 1984.

[9] R. L. de Queiroz and K. R. Rao, "Human visual sensitivity-weighted progressive image transmission using the lapped orthogonal transform," J. Electronic Imaging, vol. 1, pp. 328-338, July 1992.

[10] T. G. Lane, "Independent JPEG Group compression software," Public-domain software. 

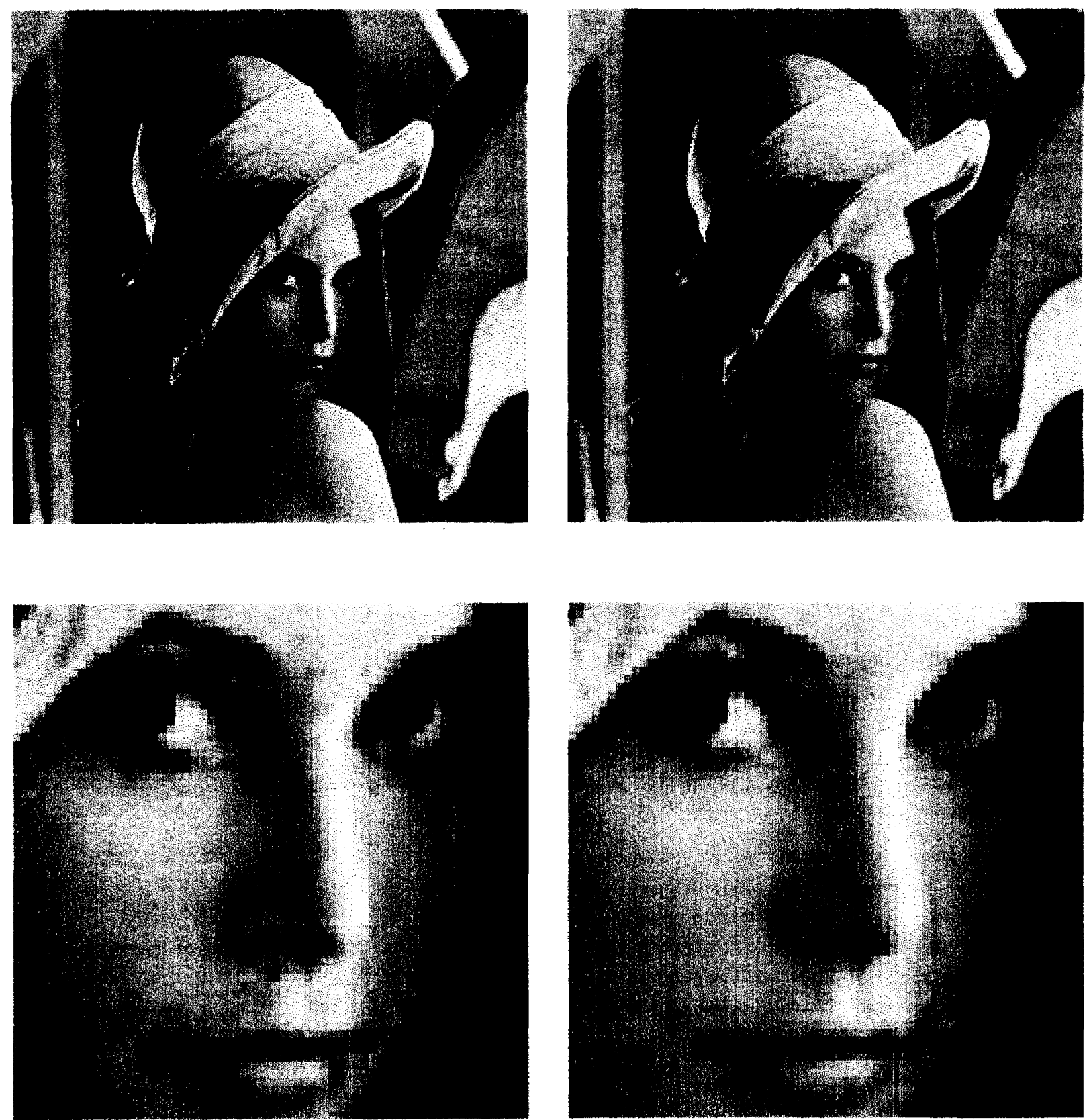

Figure 2: Examples of adaptive transform coding of the $256 \times 256$ "Lena" image, original with 8 bits/pixel. Top left: reconstructed at $0.7 \mathrm{bits} /$ pixel with the standard JPEG coder, $\mathrm{SNR}=17.7 \mathrm{~dB}$; top-right: reconstructed at 0.7 bits/pixel with the ICS coder, SNR $=19.7 \mathrm{~dB}$; bottom left: zoomed portion of the JPEG-coded image; bottom right: zoomed portion of the ICS-coded image. 